Article

\title{
Conditional Cooperation and the Marginal per Capita Return in Public Good Games
}

\section{Edward J. Cartwright * and Denise Lovett}

School of Economics, University of Kent, Canterbury, Kent CT2 7NP, UK; E-Mail: dc120@kent.ac.uk

* Author to whom correspondence should be addressed; E-Mail: E.J.Cartwright@kent.ac.uk; Tel.: +44-1227-823460; Fax: +44-1227-827850.

External Editor: Ulrich Berger

Received: 13 August 2014; in revised form: 25 October 2014 / Accepted: 31 October 2014 /

Published: 14 November 2014

\begin{abstract}
We investigate experimentally whether the extent of conditional cooperation in public good games depends on the marginal per capita return (MPCR) to the public good and type of game. The MPCR is varied from 0.2 to 0.4 to 0.8 . The 'standard' game, in which three players contribute before a follower, is compared with a leader-follower game, in which one player leads and three follow. Even though we observe less conditional cooperation for an MPCR of 0.2 , the prevalence of conditional cooperation remains relatively stable to changes in the MPCR and game timing. In contrast, the level of MPCR has a strong effect on unconditional contributions. Our results highlight the critical role played by leaders in a public good game.
\end{abstract}

Keywords: public good; conditional cooperation; reciprocity; leadership

JEL Categories: C72; H41.

\section{Introduction}

With a simple and elegant public good experiment, Fischbacher, Gächter and Fehr [1] demonstrated the prevalence of conditional cooperation. They found that around half of their subjects could be classified as conditional cooperators, in the sense that own contribution was an increasing function of the average contribution of others. The findings of Fischbacher et al. [1] have proved robust to different 
contexts and countries [2-4]. They are also of wide applied interest; conditional cooperation can help us understand, for example, dynamic changes in public good contributions (e.g., [5,6]). Open questions remain, however, about the extent and implications of conditional cooperation. In this paper, we shall address three inter-related issues that have not been previously considered in the literature. We introduce each issue in turn.

Issue 1: In the original Fischbacher et al. [1] study the marginal per capita return (MPCR) from contributing to the public good was 0.4. Subsequent studies have considered different values of the MPCR, and individual heterogeneity in the return, but only within the narrow range of 0.3 to $0.6[7,8]$. No study on conditional cooperation, as far as we are aware, has systematically varied the MPCR. We know that unconditional contributions in public good games vary with the MPCR (e.g., [9-11]). It seems, therefore, plausible that the extent of conditional contribution may also vary. The main contribution of the current paper is to address this issue.

In Section 3 of the paper (and an Appendix) we apply the Falk and Fishchbacher [12] theory of reciprocity and Fehr and Schmidt [13] model of inequality aversion to explore the role of the MPCR. We demonstrate two distinct reasons why the MPCR may influence conditional cooperation. First, there may be a critical value of the MPCR below which a person is not conditionally cooperative and above which they are. For example, a person may behave as a free-rider if the MPCR is 0.4 but behave as a conditional cooperator if the MPCR is 0.8 . Second, the extent to which a conditional cooperator 'matches' the average contribution of others may be increasing in the MPCR. For example, a person might contribute 50 percent of the average when the MPCR is 0.4 compared to 100 percent when the return is 0.8 .

In Section 5 of the paper, we report on an experiment where the MPCR is varied between the relatively extreme values of $0.2,0.4$ and 0.8 . This allows us to explore whether conditional cooperation systematically depends on the MPCR. We find evidence for both types of influence discussed in the previous paragraph. In particular, the prevalence of conditional cooperation is reduced when the MPCR is 0.2 . One could argue, however, that the differences are relatively small. For instance, conditional cooperation is the modal behavioral type even with an MPCR of 0.2 . Our interpretation of the experimental results will, therefore, be that the extent of conditional cooperation is relatively stable across different values of the MPCR.

Issue 2: The original Fischbacher et al. [1] study considered a game in which three people make an unconditional contribution before a fourth person makes a contribution conditional on the average of the earlier three. In application it is arguably more interesting to consider an alternative game in which one player leads by example (e.g., [14-16]). That is, to consider a game where one person makes an unconditional contribution before three people (or some other number) make a contribution conditional on the leader contribution. There are important theoretical differences between these two types of game. For example, in Section 3 we shall show that less conditional cooperation may be expected in a leader-follower game than the original game. Such differences would be vital to take account of when applying results on conditional cooperation.

Our experiment design allows us to compare behavior in a leader-follower game with that in the original game use by Fischbacher et al. [1]. We find that the type of game makes little difference to behavior. This is a reassuring result in terms of application, because it suggests that conditional cooperation is not overly dependent on specific details of the strategic environment. It does, however, 
raise interesting questions about how subjects reason though such games. We shall argue that our results are consistent with a 'two player' reasoning process in which 'everyone else in the game' is collapsed into a unique player [17]. ${ }^{1}$ In a two player context, the leader-follower game and the original game used by Fischbacher et al. [1] become equivalent.

Issue 3: The literature has primarily focused on conditional contributions, i.e., the contributions of 'followers'. The relationship between unconditional, or leader contributions, and conditional contributions remains relatively unexplored. This, however, can inform on the underlying motivations behind a positive contribution. To illustrate the issue, suppose that a person knows to expect conditional cooperation from others. This may incentivize her to make a large unconditional contribution [18]. Hence a 'free-rider' may make a large leader contribution. At the opposite end of the scale a conditional cooperator may contribute zero in the position of leader: If others contribute, a conditional cooperator is willing to reciprocate; this does not tell us he will contribute in the position of leader, or that he expects others to contribute.

The possibility of strategic leadership has been mentioned in the literature (e.g., [14,19,20]). To fully explore the possibility, however, it is necessary to have relatively extreme values of the MPCR and a leader-follower game (more on this below). In exploring issues 1 and 2 discussed above we are, therefore, naturally able to explore issue 3. Our experimental results show significant effects. In particular, we find that the unconditional contribution is far more sensitive to the MPCR and type of game than the conditional contribution. This sensitivity feeds through into a large effect on aggregate outcomes. We find, though, little evidence of strategic behavior. For example, with a high MPCR it was optimal for a leader to contribute his full endowment but free-riders did not exploit this opportunity. Our results are consistent with those of Gächter et al. [19] who find that more cooperative followers contribute more as leaders. They are also consistent with those of Leipold et al. [21] who find that cooperative individuals are better at predicting the types of others. The distinguishing aspect of our results is that we obtain them in a more 'extreme' environment where the potential gains to strategic behavior are higher.

The rest of the paper proceeds as follows. In Section 2 we introduce the linear public good games we shall study. In Section 3 we provide some theoretical predictions. In Section 4 we describe our experimental design. In Section 5 we present our experimental results. In Section 6 we conclude. An Appendix contains more detail on the theoretical predictions.

\section{Linear Public Good Game}

We shall consider variants on the standard linear public good game. There are $n$ members of a group who are each endowed with $E$ units of a private good. Independently of each other they must decide how much of their endowment to contribute towards a public good. Let $x_{i} \in[0, E]$ denote the contribution of member $i$ and let $X=\sum_{i} x_{i}$ denote total contributions. Total contributions to the public good are multiplied by factor $M>0$ and split evenly amongst group members. Let $m=M / n$ denote the marginal per capita return (MPCR) on the public good. The final 'monetary' payoff of member $i$ is given by

1 The idea of a 'representative' other player is not new. See, for example, chapter 7 of Camerer [17]. 


$$
u_{i}\left(x_{1}, \ldots, x_{n}\right)=E-x_{i}+m X
$$

We consider two variants of the linear public good game that differ in the timing of decisions.

In a leader-follower game the timing is as follows: Member 1 decides how much to contribute to the public good. Having observed the contribution of member 1, group members 2 to $n$ simultaneously and independently of each other decide how much to contribute. The followers (members 2 to $n$ ) can, thus, make their contribution conditional on the contribution of the leader (member 1).

In a follower-average game the timing is as follows: Members 1 to $n-1$ decide simultaneously and independently of each other how much to contribute to the public good. Having observed the mean average contribution of members 1 to $n-1$, group member $n$ decides how much to contribute. The follower (member $n$ ) can, thus, make her contribution conditional on the average contribution of the leaders (members 1 to $n-1$ ).

In both the leader-follower and follower-average game the strategy set of leaders and followers are identical. Leaders make an unconditional contribution and so a strategy simply consists of a contribution from set $[0, E]$. In explaining the strategy set of a follower, let $L$ denote the average leader contribution. This is given by the contribution of the leader in a leader-follower game or the average contribution of leaders in a follower-average game. Followers make a conditional contribution and so a strategy consists of a function $c$ mapping from $[0, E]$ to $[0, E]$. In interpretation $c_{i}(L)$ is the contribution player $i$ will make if he is a follower and the average leader contribution is $L$. In experiments the value of $L$ is typically rounded to the nearest integer. A strategy can then by represented by a contribution table with the $E+1$ possible values of average leader contribution in one column and the followers contribution in a second column.

We shall refer to a particular game as $\Gamma(Y, m, n, E)$ where $Y$ details if the game if a follower-average or leader-follower game. In the following we shall be interested in the comparative statics of changes in game timing, $Y$, and MPCR, $m$.

\section{Theory}

If $M<n$ then a member maximizes his own monetary payoff by contributing zero to the public good. This familiar logic motivates the simple prediction that every member of the group will contribute zero to the public good if $m<1 .^{2}$ A positive contribution does, however, generate a positive externality for other group members. Specifically, if $M>1$ then it is socially efficient for a group member to contribute his entire endowment $E$. This can motivate an alternative prediction, namely that every player contributes $E$ to the public good if $m>1 / n$ and contributes zero otherwise.

According to these most basic of predictions, the size of the MPCR should only influence choice around the $m=1 / n$ and $m=1$ critical values. Whether the game is a leader-follower or follower-average game should make no difference. Neither should it matter whether the member is a leader or follower. In the remainder of this section we motivate some basic hypotheses as to why the size of MPCR and type of game may make a more substantial difference. We consider in turn the role of followers and leaders.

2 Everyone contributing 0 is the unique Nash equilibrium of both the leader-follower and follower-average game. 
In order to motivate our hypotheses we shall draw on two of the leading models of social preferences. Specifically, in an Appendix to this paper we work through in detail the consequences of inequality aversion using the model of Fehr and Schmidt [13]. This will be complemented by discussing a result due to Falk and Fischbacher [12] that details the consequences of sequential reciprocity. Clearly, there are other models of social preferences we could consider. Both of these models, however, are frequently used as explanations for conditional cooperation and our primary objective in this section is to demonstrate that the MPCR and game timing could reasonably be expected to influence behavior in a systematic way.

\subsection{Conditional Cooperation}

The existing evidence suggests that a large proportion of followers will behave in a conditionally cooperative way. Conditional cooperation comes, however, in different guises and so we introduce some terminology. We shall say that member $i$ is a strong conditional cooperator in game $\Gamma$ if $c_{i}(L)=L$ for all $L$. In other words, member $i$ is a strong conditional cooperator if he exactly matches the average leader contribution. We say that member $i$ is a weak conditional cooperator in game $\Gamma$ if $d c_{i}(L) / d L>0$ but $c_{i}(L)<L$ for some $L>0$. Therefore, member $i$ 's contribution is increasing in the average leader contribution but potentially less than the leader contribution (Fischbacher and Gächter [6]). Finally, we say that member $i$ is a free- rider if $c_{i}(L)=0$ for all $L$.

It is useful for us to capture the distinction between strong and weak cooperation and free-riding. Thus, we introduce cooperation factor, $\theta_{i}=\operatorname{covar}\left(L, c_{i}(L)\right) / \operatorname{var}(L)$ as a measure of the extent of conditional cooperation of member $i$. If member $i$ is a strong conditional cooperator then $\theta_{i}=1$ and if she is a free-rider $\theta_{i}=0$. More generally, the higher is $\theta_{i}$ then the higher the extent of conditional cooperation. It is important to clarify that we characterize members as strong or weak cooperators or free-riders taking a particular game $\Gamma$ as given. To capture this we will, where appropriate, write $\theta_{i}(\Gamma)$ to acknowledge that the cooperation factor is game specific.

Let us focus first on the follower-average game and the Falk and Fischbacher [12] theory of sequential reciprocity. Proposition 11 of Falk and Fischbacher (see their online Appendix) shows that if the follower believes the $n-1$ leaders each contributed $L$ then she will contribute ${ }^{3}$

$$
c_{n}(L)=\max \left\{0, L-\frac{1-m}{\rho_{n} m(n-1)}\right\}
$$

where $\rho_{n}$ measures member $n$ 's level of reciprocity. The basic intuition behind this result is that a positive leader contribution is intentional kindness and so the follower will want to reciprocate with a positive contribution of her own. Equation (2) gives some clear predictions on the extent of conditional cooperation. For instance, only weak (not strong) conditional cooperation is predicted. In evaluating the role of the MPCR we highlight that the value of $\rho_{n}$ should not be game specific. If, therefore, $\rho_{n}>0$ we get $\theta_{n}(\Gamma)>0$ for any game $\Gamma .{ }^{4}$ Conversely, if $\rho_{n}=0$ we get $\theta_{n}(\Gamma)=0$ for any game $\Gamma$. This leads to our first hypothesis.

\footnotetext{
If $\rho_{n}=0$ then set $c_{n}(L)=0$.

4 This claim is only true if $c_{n}(E)>0$. But, this will be the case except for relatively extreme parameter values. For instance, the claim is valid for the games used in our experiments.
} 
Hypothesis 1: (a) The proportion of conditional cooperators is independent of the MPCR. (b) The cooperation factor of conditional cooperators is increasing in the MPCR.

Hypothesis 1 suggests that changes in the MPCR influence the extent of weak cooperation but do not switch a member between cooperation and free-riding. This hypothesis will now be contrasted with a prediction derived from the Fehr and Schmidt [13] model of inequality aversion. In the Appendix, we demonstrate that if the follower believes the $n-1$ leaders each contributed $L$ then she will contribute $L$ if $\beta_{n}+m>1$ and contribute 0 otherwise, where $\beta_{n} \leq 1$ measures member $n$ 's aversion to earning more than others. Therefore, the follower is either a strong conditional cooperator or a free-rider. Moreover, there is predicted to be some critical value of the MPCR $m_{n}^{*}=1-\beta_{n}$ above which she will be a conditional cooperator. Because $\beta_{n}$ can take any value between zero and one the critical value of the MPCR can also take any value between zero and one. Different members are likely to have a different critical value. This leads to our second hypothesis.

Hypothesis 2: (a) The proportion of conditional cooperators is increasing in the MPCR. (b) The cooperation factor of conditional cooperators does not depend on the MPCR.

In comparing hypotheses 1 and 2 we see quite distinct ways in which the MPCR can influence conditional cooperation. We will not be pushing this as a way to distinguish between competing models of social preferences. Hypothesis 1 could, for example, be derived from a non-linear model of inequality aversion (e.g., [22]). The point is more to show that changes in the MPCR can plausibly have very different effects on behavior. An increase in the MPCR could increase the extent of conditional cooperation of existing cooperators and/or switch members from free-riders to conditional cooperators. Hypotheses 1 and 2 also suggest the previously distinguished critical values of $m=1 / n$ and $m=1$ lose their importance. Indeed, a follower who is strongly averse to inequality, $\beta_{n}>1-1 / n$, or has desire for reciprocation, $\rho_{n}>0$, is predicted to be a conditional cooperator even if the MPCR is below the level at which it is socially efficient to contribute to the public good. We shall discuss this possibility more fully in Section 5 .

Let us now briefly turn our attention to a leader-follower game. This game is distinguished from the follower-average game by less information. Specifically, in a follower-average game the follower knows the total contribution of all other group members. In a leader-follower game a follower only knows the contribution of one other group member. This means that beliefs about the contributions of others become important, and there are various assumptions one could make (see the Appendix). For plausible assumptions it is simple to obtain direct analogs of hypotheses 1 and 2. More relevant is that a clear difference between the leader-follower and follower-average game shows up. This difference is captured in our next hypothesis.

Hypothesis 3: The average cooperation factor is, ceteris paribus, lower in a leader-follower game than follower-average game.

The intuition behind Hypothesis 3 follows directly from the lack of information in a leader-follower game. In particular, in a leader-follower game, a follower knows only that the leader has been kind or that the leader will earn a relatively low payoff. The kindness and relative payoff of others is unknown. 
This uncertainty means a follower is less willing to contribute. ${ }^{5}$ For instance, with the Fehr and Schmidt [13] model we see that a follower may want to only contribute a fraction of the leader contribution, $c_{i}(L)=\delta L$ for all $L$ where $\delta<1$. This can be compared with the strong cooperation predicted in the follower-average game.

\subsection{Leader Contribution}

We turn next to the contribution of a leader. Crucial here are the beliefs of a leader and, in particular, whether a leader expects conditional cooperation. In the model of Falk and Fischbacher [12] it is assumed that the reciprocity parameters, $\rho_{1}$ to $\rho_{n}$, are common knowledge. This means that a leader must be aware that followers may reciprocate. Fehr and Schmidt [13] make the weaker assumption that each person has some prior beliefs about the inequality aversion of others. The clear suggestion, however, is again that a leader should expect the possible presence of conditional cooperators.

To see the implications of this consider, first of all, a purely 'selfish' leader in the leader-follower game. Suppose, the leader expects that each follower is, independently, a strong conditional cooperator with probability $p$ and a free-rider with probability $1-p$. Then the expected payoff of the leader if she chooses $L$ can be written

$$
U_{1}(L)=E-L+m(L+p(n-1) L)
$$

Thus, the leader maximizes her payoff by contributing the full endowment $E$ if

$$
m>\frac{1}{1+p(n-1)}
$$

She has an incentive to contribute because she recognizes her contribution can have a positive effect on the contributions of others.

This picture is highly stylized in that we have ignored the possibility that weak conditional cooperation or that the leader is not selfish. The general picture, however, is clear enough and summarized in our next hypothesis.

Hypothesis 4: There exists a critical value of the MPCR above which the leader has an incentive to contribute the full endowment towards the public good.

This critical value will depend on beliefs about others, and on the type of game. We shall come back to this point shortly. Before doing that we want to highlight the critical value of $m$ need not be too extreme. For instance, in a 'standard' setting where $n=4$ and $p \approx 0.5$ we get an estimated critical value of $m=0.4$.

Let us now contrast the leader-follower game with follower-average game. In a leader-follower game the leader has the chance to influence $n-1$ followers while in a follower-average game she can only influence one follower. This makes a big difference to incentives. To illustrate this point we note that in

5 The only exception is if a follower believes other followers will give more than the leader. One could, for instance, obtain an equilibrium where the leader contributes 0 but followers contribute a positive amount because they believe other followers will contribute a positive amount. Hypothesis 3 is based on the assumption that a follower believes other followers will contribute (weakly) less than the leader. 
a follower-average game the difference in expected payoff for a selfish player from contributing $E$ rather than 0 can be written

$$
U_{i}(E)-U_{i}(0)=m E\left(1+\frac{p}{n-1}\right)-E
$$

Thus, the leader gains by contributing $E$ if

$$
m>\frac{1}{1+\frac{p}{n-1}}
$$

A critical value for $m$ still exists but at a much higher level.

Hypothesis 5: The critical value above which a leader has an incentive to contribute is higher in a follower-average game than leader-follower game.

And note that the critical value of $m$ does now take relatively extreme values. For instance, in the 'standard' setting where $n=4$ and $p \approx 0.5$ we get an estimated critical value of $m=0.86$.

The final issue we address is that of a 'non-selfish' leader. Under quite mild conditions one obtains the following prediction from either the Fehr and Schmidt [13] or Falk and Fischbacher [12] models.

Hypothesis 6: The critical value above which a leader has an incentive to contribute is higher for a conditional cooperator than free-rider.

In other words, conditional cooperators are 'less willing' to contribute as leaders. This prediction may be surprising. The intuition, however, is straightforward. To explain consider a leader-follower game. If the leader contributes 0 then all the followers will contribute 0 meaning no inequality and (in the terminology of Falk and Fischbacher [12]) no unkindness. If the leader makes a positive contribution then inequality and intentional unkindness are almost inevitable. ${ }^{6}$ A non-selfish leader has to, therefore, trade off gains from the public good with a lower social payoff. A selfish player focuses only on the returns from the public good.

Hypothesis 6 suggests a potential 'mismatch' between conditional and unconditional contribution. For example, someone who is not inequality averse may make a positive contribution as a leader but not as follower. Conversely, someone who is highly inequality averse may make a positive contribution as follower but not as leader.

\section{Experimental Design}

We use the benchmark game of Fischbacher et al. [1] as our starting point. This benchmark is a follower-average game where $n=4, E=20$ and $m=0.4$. The benchmark game is compared with games where the MPCR is 0.2 or 0.8 and/or the game is a leader-follower game. This allows us to evaluate the consequences of the MPCR and the type of game.

To help explain the experimental design in more detail consider Table 1. As you can see each experimental session consisted of three distinct parts. In the first part of session 1 subjects played the benchmark game. In the second part of the session subjects played a follower-average game with the

6 In the Falk and Fischbacher [12] model of sequential reciprocity the leader can anticipate intentional unkindness from contributing a positive amount. This lessens the incentive to contribute the stronger are her reciprocal preferences. 
higher MPCR of 0.8, and in part 3 they played a follower-average game with the lower MPCR of 0.2. Session 2 differed from session 1 in the type of game. Sessions 3 and 4 differed in the sequence with which MPCRs were used. This design allows a between subject comparison on the influence of the type of game and a within and between subject comparison on the influence of the MPCR.

Table 1. Experimental design.

\begin{tabular}{cccccc}
\hline Session & Type of Game & Part 1 & Part 2 & Part 3 & Subjects \\
\hline 1 & Follower-average & $m=0.4$ & $m=0.8$ & $m=0.2$ & 31 \\
2 & Leader-follower & $m=0.4$ & $m=0.8$ & $m=0.2$ & 25 \\
3 & Leader-follower & $m=0.2$ & $m=0.4$ & $m=0.8$ & 19 \\
4 & Leader-follower & $m=0.8$ & $m=0.2$ & $m=0.4$ & 21 \\
\hline
\end{tabular}

In motivating the experimental design we highlight that the MPCR is compared across a deliberately large range. This will allow us to explore all the six hypotheses in Section 3. For instance, an MPCR of 0.8 is above the critical value at which the leader has an incentive to contribute in a leader-follower game (for reasonable estimates of $p$ ). With an MPCR of 0.2 the leader can have no incentive to contribute (whatever her estimate of $p$ ). Similarly, an MPCR of 0.2 allows us to see whether followers are conditionally cooperative even though it is socially inefficient to contribute to the public good.

The strategy method was used throughout the experiment (as is standard in this literature on conditional cooperation, e.g., Fischbacher et al. [1]). Therefore, in each of the three parts of a session a subject was asked to (i) choose an unconditional contribution; and then (ii) fill in a contribution table indicating what contribution they would make for any possible average leader contribution. (The full instructions are contained in the supplementary material.) The strategy method allows us to measure the extent of conditional cooperation and to see what a subject would do in the position of both leader and follower.

Intuitively, one might expect the strategy method to elicit different choices from a direct response method wherein the game is played in 'real time'. The evidence, however, points otherwise for the type of games considered here (Brandts and Charness [23]). For instance, Fischbacher, Gächter and Quercia [24] compare the strategy method and direct response method in public good games and find the two yield qualitatively similar results. Similarly Brandts and Charness [25] find no difference between the two methods in sequential games. We do not, therefore, feel that use of the strategy method will unduly influence our results.

One final aspect of the design we want to emphasize is that subjects were given no feedback on their payoff or the contributions of others until the end of a session. More precisely, at the beginning of the session subjects were given the instructions to part 1 (and given general guidance the experiment would consist of three parts). After making their choices for part 1 they were given the instructions to part 2 and after making their choices for part 2 they were given the instructions to part 3. Only after making their choices for part 3 were subjects randomly allocated to groups and given feedback. ${ }^{7}$ That no feedback was given until the end of the session means that the choices of each subject can be treated as

7 See Fischbacher, Schudy and Teyssier [7] for a similar approach. Subjects were given feedback on all three parts of the experiment. 
an independent unit of observation. It also means that potential order effects, from playing three games in a row, should be diminished. We will return to this point in the results section.

A total of 96 subjects took part in the experiment recruited from across the University of Kent. The experiment took place in a computer lab using the software z-Tree [26]. The instructions were kept as close as possible to those used in previous studies and the language was deliberately neutral. Each session took around $30 \mathrm{~min}$ and the average payment was $£ 8.64$.

\section{Experimental Results}

We begin by noting that the results in the benchmark game (specific details shortly) were entirely in keeping with those observed in previous studies. We shall, therefore, focus on the effect of the MPCR and type of game. We consider in turn conditional and unconditional contributions. Before doing that an aggregate picture of outcomes may be useful to preview our main findings. Figure 1 details the expected total contribution towards the public good, given the strategies of subjects, for each of the six games. ${ }^{8}$ The portion of this contribution due to leaders and followers is also distinguished.

Sizeable differences across the six games are clearly apparent in Figure 1 with expected contributions ranging from as low 9 to as high as 37 (relative to a maximum possible of 80). Total contributions are significantly increasing in the MPCR for both the leader-follower and follower-average game $(p<0.01$ for all pairwise comparisons, bootstrapping). Total contributions are also significantly higher in the follower-average game compared to the leader-follower game when the MPCR is 0.4 or $0.8(p<0.01)$. This latter difference owes more, however, to there being three leaders than to differences in strategy. This will become clear as we look at individual strategy in more detail.

Figure 1. Average total contributions in each of the six games distinguishing the role played by leaders and followers.

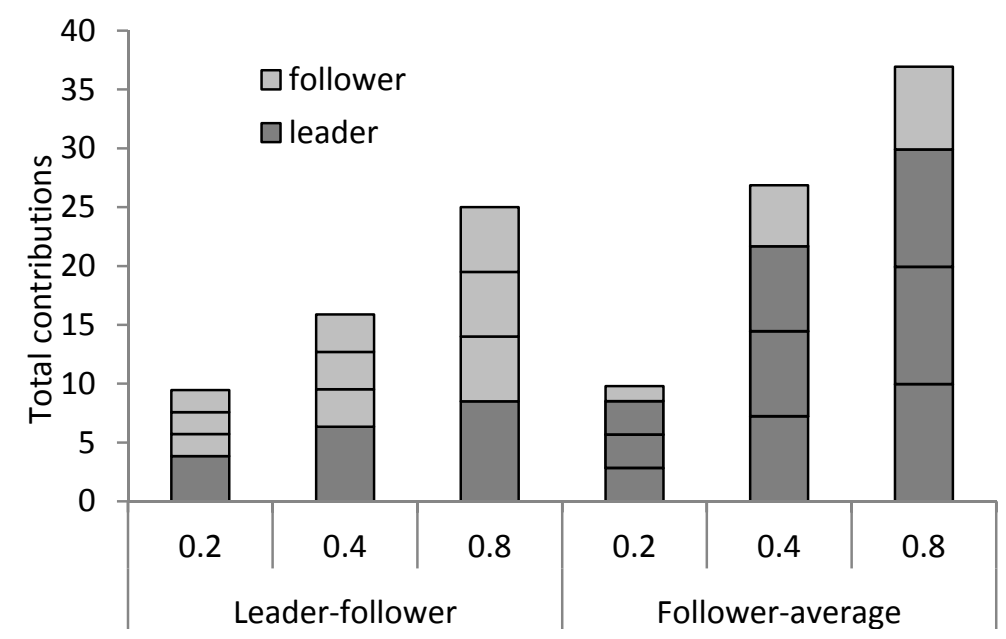

8 To explain how this is calculated consider the follower-average game. If we randomly select three subjects and take the average of their unconditional contribution we have the average leader contribution. If we randomly select a fourth subject and look at their contribution table we have the follower contribution. Combining these gives the total contribution. Repeating this exercise for all possible permutations of subjects gives the expected total contribution. Data from all parts of a session is used in deriving Figure 1. 


\subsection{Follower Behavior}

For each of the three games that a subject played they are classified as one of four behavioral types according to how they filled in the contribution table. The types are conditional-cooperator, free-rider, hump-shaped contributor, or other. Subjects are classified following the approach of Fischbacher et al. [1]. Therefore, a conditional cooperator has a Spearman rank correlation coefficient between own and average leader contribution significant at the one percent level. ${ }^{9}$ A free-rider contributes zero irrespective of average leader contribution. A hump-shaped contributor has positive Spearman rank correlation coefficient for low levels of average leader contribution and a negative coefficient for high levels of average leader contribution. We reiterate that a subject is classified for each of the three games they play. The same subject could, therefore, be classified as a conditional cooperator in one game, free-rider in another, and so on.

\subsubsection{Proportion of Behavior Types}

Table 2 summarizes the proportion of subjects of each behavioral type for the six different games considered. It provides data for all parts and for part 1 of each session only. Before going any further we note that the proportion of conditional cooperators in the leader-follower game was significantly different in part 1 of a session from parts 2 and 3 for an MPCR of 0.4 and 0.8 ( $p=0.06$ and 0.01 respectively, proportions test). ${ }^{10}$ There is, therefore, a non-negligible order effect from subjects playing three games in a row. In interpreting this effect, we reiterate that subjects were given no feedback on the outcome of part 1 (or part 2) until the end of the session. Our preferred interpretation, therefore, is that the order effect reflects subjects 'learning' from repetition (see [27]). ${ }^{11}$ This would motivate the use of data from all parts of a session and so that is the approach we shall take. ${ }^{11}$ For completeness, however, we will continue to provide the data from part 1 for comparison. Test statistics for part 1 data will be reported if and only if they substantially differ from those obtained using all the data. Crucially, none of our conclusions will depend on whether we use the data from part 1 or from all parts of a session.

Let us now look in more detail at the numbers in Table 2. The proportion of conditional cooperators is significantly lower when the MPCR is 0.2 compared to 0.4 for both the leader-follower game and follower-average game ( $p=0.08$ and $p=0.02$ respectively, proportion test). The proportion is also significantly lower when the MPCR is 0.2 compared to 0.8 for the follower-average game $(p=0.02)$. All other pairwise comparisons, however, are insignificant. Therefore, there is no significant difference between the follower-average game and leader-follower game for a fixed MPCR ( $p>0.1$, proportion test). Neither is there any difference between an MPCR of 0.4 and $0.8(p>0.3)$ or of 0.2 and 0.8 in the leader-follower game $(p=0.48)$. Overall, therefore, the proportion of conditional cooperators is relatively stable across the six games. Indeed, the proportion remains high even when the MPCR is 0.2.

9 This criterion is different from that introduced in Section 3 but clearly in the same spirit.

10 A similar order effect was observed by Fischbacher, Schudy and Teyssier [7].

11 An alternative explanation is that choices in parts 2 and 3 were influenced by the game played in part 1 . We shall see, however, that there was a basic shift towards lower contributions in parts 2 and 3 irrespective of the game played in part 1. This does not suggest a priming effect. Moreover, 'learning' effects have been observed from playing the same game many times with no feedback (e.g., [27]). 
Table 2. Proportion of subjects of each behavioral type for the six different games.

\begin{tabular}{|c|c|c|c|c|c|c|}
\hline \multirow[b]{2}{*}{ All Parts } & \multicolumn{3}{|c|}{ Leader-Follower Game } & \multicolumn{3}{|c|}{ Follower-Average Game } \\
\hline & $m=0.2$ & $m=0.4$ & $m=0.8$ & $m=0.2$ & $m=0.4$ & $m=0.8$ \\
\hline Conditional Cooperator & 47.69 & 63.08 & 53.85 & 41.94 & 70.97 & 70.97 \\
\hline Free-rider & 30.77 & 15.38 & 18.46 & 41.94 & 6.45 & 9.68 \\
\hline Hump-shaped & 3.08 & 3.08 & 6.15 & 0.00 & 3.23 & 3.23 \\
\hline Other & 18.46 & 18.46 & 21.54 & 16.13 & 19.35 & 16.13 \\
\hline \multicolumn{7}{|l|}{ Part 1} \\
\hline Conditional Cooperator & 47.37 & 72.00 & 66.67 & & 70.97 & \\
\hline Free-rider & 21.05 & 12.00 & 0.00 & & 6.45 & \\
\hline Hump-shaped & 5.26 & 4.00 & 4.76 & & 3.23 & \\
\hline Other & 26.32 & 12.00 & 28.57 & & 19.35 & \\
\hline
\end{tabular}

Given that contributing to the public good is socially inefficient when the MPCR is 0.2 it may seem counter-intuitive to observe conditional cooperation. Therefore, let us briefly comment on this (and also remind that the issue was partially covered in Section 3). The main thing to highlight is that it is not in a follower's material interests to contribute to the public good whether the MPCR is $0.2,0.4$ or 0.8 . Any positive contribution must, therefore, reflect a 'mistake', a desire to reduce inequality, to reciprocate, to conform, or similar. Only in the special case of efficiency seeking preferences (which can easily be ruled out) will it be of any relevance whether or not contributing is socially efficient. ${ }^{12,13}$ In particular, if the three leaders in a follower-average game make a positive contribution then they have benefited the follower: for every unit they contributed she gets 0.2 . It is perfectly reasonable that she would want to 'return the favor' and contribute herself. ${ }^{14}$ This can help explain why conditional cooperation remains the most common behavior type for an MPCR of 0.2. Whether or not leaders make a positive contribution for such a low MPCR is a question we shall address shortly.

Table 3 provides the results of three probit regressions in which the dependent variable is whether a subject was classified as a conditional cooperator. The results presented in the first column use data from part 1 of a session and those presented in the second and third column use the data from all parts (with a panel regression). ${ }^{15}$ We see further evidence of less conditional cooperation when the MPCR is 0.2 (the two 0.2 coefficients are jointly significant, $p=0.03$ F-test, for the second all parts regression). We also see further evidence of an order effect with conditional cooperation decreasing in parts 2 and 3 of a

12 An efficiency seeking follower will contribute a positive amount irrespective of the average leader contribution when the MPCR is 0.4 or 0.8 . None of our subjects did that. Neither did any of those in Fischbacher et al. [10]. Note that prior evidence of efficiency seeking preferences (e.g., [28]) is in a context where efficiency can be enhanced without sacrifice in own material payoff.

13 The MPCR reflects the 'price' of making a mistake, reducing inequality, reciprocating etc. So, a decrease in the MPCR may influence, say, the decision to reciprocate. But, there is no 'discontinuity' around the MPCR at which contributing becomes socially efficient, $\mathrm{m}=0.25$ in our case. This is captured in the analysis of Section 3 .

14 It is also reasonable that she would have preferred the leaders to contribute zero in order to avoid a costly round of 'gift giving'!

15 Recall that in part 1 we only have data for the leader-follower game with an MPCR of 0.4. 
session. ${ }^{16}$ Surprisingly, we see a negative coefficient for an MPCR of 0.8 in the leader-follower game; this, though, may also be picking up an order effect. ${ }^{17}$

As a final piece of evidence regarding the proportion of conditional cooperators we can exploit the fact that each subject is observed playing a game for three different values of the MPCR. In total 34 of the 96 subjects were conditional cooperators in all three games (of which 5 were strong conditional cooperators in all three games). 16 subjects were conditional cooperators for an MPCR of 0.4 and 0.8 but not for a return of 0.2 (of which 3 went from free-riding to strong conditional cooperation). ${ }^{18}$ These are the most common and second most common patterns of behavior. To put these numbers in perspective suppose that subjects were to independently decide in each part whether or not to be conditional cooperators. Then we would not expect 33 subjects to be conditional cooperators in all three games $(p<0.001)$. However, we could expect that 16 subjects would only be conditional cooperators for a return of 0.4 and $0.8(p=0.26)$.

Table 3. Results of two probit regressions with conditional cooperator as dependent variable, using the data from part 1 or the full sample. A panel regression is used for all parts.

\begin{tabular}{cccc}
\hline & Part 1 & All Parts & All Parts \\
\hline Constant & $0.552 *(0.238)$ & $1.173 * *(0.416)$ & $1.068 * *(0.476)$ \\
0.2 Return & $-0.649 *(0.392)$ & $-0.709 * * *(0.272)$ & $-0.839(0.602)$ \\
0.8 Return & $-0.152(0.389)$ & $-0.187(0.271)$ & $0.507(0.474)$ \\
Leader-follower Game & $0.030(0.359)$ & $-0.326(0.419)$ & $-0.095(0.560)$ \\
0.2 Return * Leader-follower & - & - & $0.139(0.638)$ \\
0.8 Return * Leader-follower & - & - & $-0.905 *(0.519)$ \\
Part 2 & - & $-0.311(0.277)$ & $-0.507 *(0.303)$ \\
Part 3 & - & $-0.647 * *(0.301)$ & $-0.613 *(0.338)$ \\
\hline Number of Observations & 96 & 288 & 288 \\
Wald $\chi^{2}$ & 3.48 & $17.45 * * *$ & $19.33 * * *$ \\
Pseudo R & 0.03 & - & - \\
\hline
\end{tabular}

Note: Standard errors in parentheses, which are robust in the Part 1 model and cluster-robust (clustering upon individual) for All Parts regression; *, **, *** denote significance at $10 \%, 5 \%$ and $1 \%$ respectively.

The basic picture seems to be that the MPCR and type of game make little difference to the proportion of conditional cooperators. There is evidence of less conditional cooperation for an MPCR of 0.2 , consistent with Hypothesis 2(a), but conditional cooperation still remains common.

\subsubsection{Cooperation Factor}

To say that a person behaves as a conditional cooperator merely means her contribution is increasing in the average leader contribution. We now question to what extent subjects matched the average leader

16 We cannot reject the null that the part 2 and part 3 coefficients are the same $(p=0.70$, F-test).

17 If we look at the net-effect then there is no significant difference between the follower-average game with an MPCR of 0.8 and leader-follower game with an MPCR of 0.4 ( $p=0.40, \mathrm{~F}$-test) but there is a difference the follower-average game with an MPCR of 0.8 and leader-follower game with an MPCR of $0.8(p=0.09)$.

18 Of the remaining subjects: 24 were a conditional cooperator for at least one game while 17 were not a conditional cooperator in any game. Only 6 subjects were classified as a free-rider in all three games. 
contribution. The cooperation factor $\theta_{i}(\Gamma)$ of each subject in each game (as defined in Section 3 ) can be found by regressing their own contribution against average leader contribution. ${ }^{19}$ Table 4 summarizes the average cooperation factor of conditional cooperators for the six games. An average cooperation factor in the region of 0.6 to 0.8 is expected for an MPCR of 0.4 ([6]) and means that conditional cooperators contribute around 60 to $80 \%$ of the average leader contribution.

Table 4. Average cooperation factor of conditional cooperators for the six games.

\begin{tabular}{ccccccc}
\hline & \multicolumn{3}{c}{ Leader-Follower Game } & \multicolumn{3}{c}{ Follower-Average Game } \\
\cline { 2 - 7 } & $\boldsymbol{m = 0 . 2}$ & $\boldsymbol{m}=\mathbf{0 . 4}$ & $\boldsymbol{m = 0 . 8}$ & $\boldsymbol{m}=\mathbf{0 . 2}$ & $\boldsymbol{m}=\mathbf{0 . 4}$ & $\boldsymbol{m}=\mathbf{0 . 8}$ \\
\hline All Parts & 0.619 & 0.633 & 0.695 & 0.509 & 0.798 & 0.834 \\
Part 1 & 0.773 & 0.642 & 0.731 & - & 0.798 & - \\
\hline
\end{tabular}

In the leader-follower game we see an average cooperation factor in the range 0.6 to 0.7 for all values of the MPCR with no significant differences $(p>0.3$, Mann-Whitney, even if we only use the data for part 1). In the follower-average game the average cooperation factor for an MPCR of 0.2 is significantly lower than for an MPCR of 0.4 and 0.8 ( $p=0.005$ and 0.002 respectively). In addition, the cooperation factor in the leader-follower game is significantly lower than in the follower-average game when the MPCR is 0.4 and 0.8 , but not 0.2 ( $p=0.07,0.08$ and 0.77 respectively, Mann-Whitney). These differences are consistent with hypotheses $1(\mathrm{~b})$ and 3.

Table 5 provides the results of four Tobit regressions in which the dependent variable is cooperation factor. For completeness we provide the results of regressions where all subjects are included and those where only conditional cooperators are included. Over the entire sample the cooperation factor is significantly lower with an MPCR of 0.2 . Our primary interest, however, is in the cooperation factor of conditional cooperators. We see that the cooperation factor is lower with an MPCR of 0.2 , higher with an MPCR of 0.8 , and lower in a leader-follower game, but none of these differences are statistically significant. We cannot rule out, therefore, the possibility that the differences observed in the raw aggregates reflect an order effect.

One could argue that we see more systematic difference in terms of cooperation factor than we did the proportion of behavior types. Overall, though, the basic picture again seems to be that the MPCR and type of game do not make much difference. Even for the low MPCR of 0.2 we have seen that conditional cooperation is the modal type of behavior and that the cooperation factor of conditional cooperators is above 0.5 . This suggests that conditional cooperation is surprisingly robust to changes in the MPCR and game timing.

19 For each subject and each game we have 21 data points from his or her completion of the contribution table (because the average leader contribution can range from 0 to $\mathrm{E}$ ). When own contribution is regressed against leader contribution (and a constant term) the cooperation factor $\theta_{i}(\Gamma)$ is given by the coefficient on leader contribution. 
Table 5. The results of Tobit regressions with cooperation factor as the dependent variable.

\begin{tabular}{ccccc}
\hline & & Part 1 & \multicolumn{2}{c}{ Entire Sample } \\
\hline Cooperation Factor & All Subjects & Conditional Cooperators & All Subjects & Conditional Cooperators \\
\hline Constant & $0.643 * * *(0.149)$ & $0.904 * * *(0.110)$ & $0.606 * * *(0.155)$ & $0.938 * * *(0.104)$ \\
0.2 Return & $-0.221(0.278)$ & $0.210(0.194)$ & $-0.483 * *(0.192)$ & $-0.180(0.116)$ \\
0.8 Return & $0.062(0.237)$ & $0.097(0.163)$ & $0.175(0.186)$ & $0.160(0.107)$ \\
Leader-follower Game & $-0.235(0.219)$ & $-0.197(0.148)$ & $-0.140(0.198)$ & $-0.149(0.130)$ \\
0.2 Return * Leader-follower & - & - & $0.241(0.217)$ & $0.167(0.134)$ \\
0.8 Return * Leader-follower & - & - & $-0.250(0.212)$ & $-0.074(0.121)$ \\
Part 2 & - & - & $-0.241 * *(0.109)$ & $-0.132 *(0.067)$ \\
Part 3 & - & - & $-0.229 * *(0.109)$ & $-0.090(0.069)$ \\
\hline F-test & 96 & 63 & 288 & 164 \\
Pseudo $R^{2}$ & 1.00 & 0.73 & $35.95 * * *$ & $20.30 * * *$ \\
\hline
\end{tabular}

Note: Cluster robust standard errors (clustering upon individual) in parentheses; $*, * * * * *$ denote significance at $10 \%, 5 \%$ and $1 \%$ respectively.

\subsection{Leader Behavior}

We turn now to the unconditional contribution. Table 6 details the average unconditional contribution for each game, also distinguishing across behavior type. An increase in the MPCR leads to a sizable increase in unconditional contributions from around a seventh to one half of the endowment. In the leader-follower game the leader contribution with an MPCR of 0.8 is significantly higher than that with an MPCR of 0.4 ( $p=0.09$, Mann Whitney), and that with an MPCR of 0.4 is significantly higher than with an MPCR of $0.2(p=0.02) .{ }^{20}$ In the follower-average game, the unconditional contribution with an MPCR of 0.8 is not significantly higher than that with a return of $0.4(p=0.13)$, but that with an MPCR of 0.4 is significantly higher than with a return of $0.2(p=0.01)$. The differences between the leader-follower and follower-average game are statistically insignificant $(p>0.3)$ unless we look only at the data from part $1(p=0.05)$.

Table 7 reports the results of three Tobit regressions in which the dependent variable is the unconditional contribution as a proportion of endowment. Consistent with the earlier results we see a strong effect due to the MPCR but only weak evidence of any effect due to game timing (the two 0.8 coefficients are jointly significant, $p=0.01 \mathrm{~F}$-test, for the second all parts regression). With an MPCR of 0.2 unconditional contributions are predicted to fall by around 24 to $40 \%$ of the endowment when compared to the baseline MPCR of 0.4. With an MPCR of 0.8 they are predicted to increase by around $15 \%$ of the endowment.

Finally, we turn our attention to differences in unconditional cooperation across behavior type. Recall (Hypothesis 6) that 'selfish' free-riders may have most incentive to make a large leader contribution. The intuition being that a large leader contribution will lead to higher contributions but also increased inequality. To make this idea more concrete we can work out what the optimal unconditional

20 The difference between a marginal return of 0.2 and 0.8 is highly significant $(p<0.01)$ for both the leader-follower and follower-average game. 
contribution would have been for someone who purely wants to maximize own payoff. ${ }^{21}$ The optimum was zero in all games except a leader-follower game with an MPCR of 0.8. In this game the optimal leader contribution was the full endowment of 20. Moreover, the potential gains were substantial with an expected payoff of 35 from contributing 20 compared to 22 from contributing zero. A 'selfish' individual has, therefore, the incentive to make a large leader contribution in this game.

Table 6. Average unconditional contribution overall and by type for the six different games.

\begin{tabular}{ccccccc}
\hline & \multicolumn{3}{c}{ Leader-Follower Game } & \multicolumn{3}{c}{ Follower-Average Game } \\
\hline All parts & $\boldsymbol{m}=\mathbf{0 . 2}$ & $\boldsymbol{m}=\mathbf{0 . 4}$ & $\boldsymbol{m}=\mathbf{0 . 8}$ & $\boldsymbol{m = 0 . 2}$ & $\boldsymbol{m}=\mathbf{0 . 4}$ & $\boldsymbol{m}=\mathbf{0 . 8}$ \\
\hline Overall average & 3.85 & 6.34 & 8.49 & 2.84 & 7.23 & 9.97 \\
Conditional cooperator & 5.13 & 6.90 & 9.31 & 3.54 & 6.68 & 11.18 \\
Free-rider & 0.35 & 2.60 & 3.25 & 2.15 & 2.50 & 1.00 \\
Hump-shaped & 7.50 & 7.50 & 8.75 & $\mathrm{n} / \mathrm{a}$ & 10.00 & 8.00 \\
Other & 5.75 & 7.33 & 10.86 & 2.80 & 10.33 & 10.40 \\
\hline Part 1 & & & & & & \\
\hline Overall average & 4.58 & 4.64 & 9.05 & & 7.23 & \\
Conditional cooperator & 5.22 & 5.22 & 9.00 & & 6.68 & \\
Free-rider & 1.50 & 0.00 & $\mathrm{n} / \mathrm{a}$ & & 2.50 & \\
Hump-shaped & 5.00 & 3.00 & 5.00 & & 10.00 & \\
Other & 5.80 & 6.33 & 9.83 & & 10.33 & \\
\hline
\end{tabular}

Table 7. The results of Tobit regressions with unconditional contribution as a proportion of total endowment as the dependent variable. A panel regression is used for all parts.

\begin{tabular}{cccc}
\hline Unconditional Contribution & Part 1 & All Parts & All Parts \\
\hline Constant & $0.318 * * *(0.097)$ & $0.142(0.099)$ & $0.176^{*}(0.104)$ \\
Conditional Cooperator & $0.031(0.091)$ & $0.232 * * *(0.065)$ & $0.230 * * *(0.066)$ \\
0.2 Return & $0.041(0.118)$ & $-0.223 * * *(0.059)$ & $-0.348 * * *(0.117)$ \\
0.8 Return & $0.340 * *(0.153)$ & $0.163 * * *(0.057)$ & $0.134(0.111)$ \\
Leader-follower Game & $-0.220 *(0.121)$ & $-0.033(0.096)$ & $-0.096(0.119)$ \\
0.2 Return * Leader-follower & - & - & $0.167(0.133)$ \\
0.8 Return * Leader-follower & - & - & $0.039(0.126)$ \\
Part 2 & - & $0.034(0.057)$ & $0.044(0.065)$ \\
Part 3 & - & $-0.024(0.058)$ & $0.016(0.066)$ \\
\hline Number of Observations & 96 & 288 & 288 \\
LR $\chi^{2}$ test/Wald $\chi^{2}$ & $8.79 *$ & $71.24 * * *$ & $73.07 * * *$ \\
Pseudo $R^{2}$ & 0.061 & & \\
\hline
\end{tabular}

Note: Standard errors in parentheses, which are robust in the Part 1 model and cluster-robust (clustering upon individual) for All Parts regression; *,**,** denote significance at $10 \%, 5 \%$ and $1 \%$ respectively.

21 That is, we look at expected payoff for all possible values of unconditional contribution given the actual conditional contributions of our subjects. 
The data in Table 6 show no evidence of subjects taking advantage of this possibility. Across the board the unconditional contribution of conditional cooperators is higher than that of free-riders. ${ }^{22}$ Indeed, in Table 7 we see, looking at the data from all parts, that conditional cooperators make a significantly higher unconditional contribution. This evidence is inconsistent with Hypothesis 6, but is consistent with the previous literature. For example, Gächter et al. [19] find that more cooperative followers make a larger leader contribution. This partly reflected a false consensus effect where cooperative leaders were more optimistic about followers. Likewise, Leipold et al. [21] find that cooperative types are better at predicting what others will do. Note, however, that we are the first to consider a setting where the selfish interest is to contribute. We are, thus, the first to explicitly test whether less cooperative types appreciate the potential gains from a high leader contribution.

Another interesting angle with which to test for strategic leadership is to compare our results with those from public good games with simultaneous choice. In a game with simultaneous choice there is no possible means to influence others and so one obtains a baseline level of 'non-strategic' contribution. With strategic leadership, in a leader-follower or follower-average game, one would expect unconditional contributions to exceed this baseline. The tentative evidence suggests they do not. For instance, Goeree et al. [11] compare, amongst other things, simultaneous four player games with an MPCR of 0.4 and 0.8. Mean contributions were 4.9 and 10.6, respectively, from an endowment of 25. Similarly, Isaac and Walker [9] and Isaac, Walker and Williams [10] compared an MPCR of 0.30 and 0.75 and found mean contributions of around 17 and 25 from an endowment of 50. These numbers are very similar to the unconditional contributions we observed.

If unconditional contributions do not exceed the baseline level obtained with simultaneous choice then one obtains the prediction that total contributions in a game with exogenous leadership should not exceed those with simultaneous choice. This prediction is not inconsistent with the somewhat ambiguous effects of leadership observed in the literature. For example, Gächter et al. [29] find lower contributions with leadership, Potters, Sefton and Vesterlund [30] and Rivas and Sutter [31] find no effect of leadership, while Güth et al. [14] find increased contributions with leadership. ${ }^{23}$ In order for leadership to be particularly effective it seems leadership needs to be endogenous [31], or come with some additional power such as the ability to exclude others [14] or to be more informed [30].

\section{Conclusions}

Our starting point for this paper was the seminal contribution of Fischbacher et al. [1] on conditional cooperation. We wished to explore three related issues: (i) Whether the extent of conditional cooperation depends on the marginal per capita return (MPCR) from contributing to the public good; (ii) Whether we observe different behavior in a leader-follower game as compared to the follower-average game considered by Fischbacher et al. [1]; (iii) The connection between an individual's conditional and unconditional contribution.

22 There was one free-rider who contributed 20 as leader when the MPCR was 0.8, but this was clearly the exception. The overall proportion of subjects making an unconditional contribution of 20 was around 6\%, 12\% and 19\% for an MPCR of $0.2,0.4$ and 0.8 respectively.

23 We focus here on the treatments of most direct relevance to us, i.e. those with exogenous leadership, symmetric returns from the public good, no exclusionary power and complete information. 
We find that the MPCR makes a significant difference to the unconditional contribution: The contribution of leaders is increasing in the value of the MPCR. In contrast, we find that changes in the MPCR make little difference to the conditional contribution: The proportion of conditional cooperators and the cooperation factor is relatively stable across different values of the MPCR, even though it is reduced for an MPCR of 0.2. In interpreting these findings we note that differences in unconditional contribution, coupled with no difference in conditional cooperation, equate to a significant overall difference in contributions. With a low level of MPCR leaders give little and so followers give little. With a high level of MPCR leaders give more and so followers give more. This difference is clearly apparent in Figure 1.

Our theoretical analysis, using two leading models of social preferences, suggested that conditional contributions should be sensitive to the MPCR and game timing. That we did not observe such differences is, therefore, a challenge to the idea that conditional cooperation is driven by inequality aversion or reciprocity. To reconcile the theory with the data would essentially require social preferences to be concentrated on extreme ends of the distribution. For instance, for people to be either strongly inequality averse or not inequality averse at all. That is plausible. Our results may suggest, however, that conditional cooperation simply reflects conformity or imitation. Such behavior would not be unreasonable given that leaders did appear to take the MPCR into account. A conditional contributor will not, for example, end up contributing much when the MPCR is low. The difficulty with a conformity or imitation interpretation is to explain why conditional contributors make larger unconditional contributions.

To reinforce these points we emphasize that each subject chose his or her conditional contribution after choosing the unconditional contribution. This would appear to rule out other possible explanations for the stability of follower behavior. For example, if our results were being driven by subjects simply not understanding the instructions, particularly regarding the socially inefficient case, MPCR equal to 0.2 , then we would not expect to see such large differences in unconditional contribution. That we see large differences in unconditional contribution but so little difference in subsequent conditional contribution shows that stability in follower behavior is not an accident. Whether follower behavior is driven by social preferences or conformity is not something we can answer here.

In terms of the comparison between a leader-follower game and follower-average game we found no significant effect on behavior. From a theoretical perspective this is a surprising result given the differences between the two games. In a leader-follower game, for example, the unconditional contribution is much more critical than in a follower-average game because the contributions of three, and not one, player are conditioned on it. One explanation for why we observe no difference is that both games are seen as a 'two player game' in which all other players are collapsed into one representative player. With only two players the leader-follower and follower-average games become identical. Such a lack of sensitivity to the number of players has been noted in other contexts such as the minimum effort game [32].

We have already noted the critical role played by leaders. If the behavior of followers is not sensitive to the MPCR or details of the game then leaders effectively determine the outcome. Arguably, however, the literature has more to say about followers than leaders. It is only in recent years that attention has begun to focus more on the leader $[14,15,18,20]$ and many questions remain unanswered. One issue we were able to explore a little in this paper is whether a 'strategic' leader can take advantage of conditional 
cooperation. Specifically, in a leader-follower game with an MPCR of 0.8 we found that it was the interests of a 'selfish' leader to contribute the full endowment towards the public good. As far as we are aware we provide the first public good experiment where such a possibility has arisen. We did not, however, find any evidence of 'free-riders' changing behavior to take advantage of the different incentives.

In order to explore the potential for strategic leadership in more detail it would seem vital to question the beliefs and motives of leaders. Volk, Thöni and Ruigrok [33] find that conditional cooperators and free-riders are characterized by different levels of agreeableness. Moreover, the level of agreeableness was found to be associated with more stable preferences over time. Interestingly, the psychology literature has connected agreeableness with leadership (e.g., Judge and Bono [34]). One explanation for this connection is that agreeableness comes with greater empathy and greater empathy allows more successful leadership. Conditional cooperators may naturally, therefore, be the 'best' leaders. This conjecture is consistent with our results. In future work we hope to explore such issues further.

\section{Acknowledgements}

Denise Lovett is funded by an ESRC scholarship. This paper has benefitted considerably from the comments and advice of three anonymous reviewers.

\section{Appendix}

We shall consider here the Fehr and Schmidt [13] model of inequality aversion. Suppose that the payoff of member $i$ is given by

$$
u_{i}^{s}\left(x_{1}, \ldots, x_{n}\right)=E-x_{i}+m X-\alpha_{i} \frac{1}{n-1} \sum_{j \neq i} \max \left\{0, x_{i}-x_{j}\right\}-\beta_{i} \frac{1}{n-1} \sum_{j \neq i} \max \left\{0, x_{j}-x_{i}\right\}
$$

where $0 \leq \beta_{i}<\alpha_{i}<1$ are individual specific parameters. The monetary payoff of member $i$ is, therefore, augmented by a social payoff that depends on how much the member's contribution, and therefore payoff, differs from that of the other group members. ${ }^{24}$

Let us first consider a follower-average game where the average leader contribution is $L$. The follower cannot discern individual contributions, because she only knows the average, but we assume she believes all leaders chose $L$. Given this assumption

$$
\frac{d u_{i}^{s}}{d x_{i}}=-1+m+\beta_{i}
$$

for all $x_{i}<L$. Thus, as discussed in the main text, she maximizes her payoff by contributing $L$ if $\beta_{i}+m>1$, and by contributing zero otherwise. This suggests that any increase in the MPCR can increase the likelihood the follower will match the average-leader contribution.

Let us next consider a leader-follower game where the leader contribution is $L$. In this case, the follower cannot discern the contributions that other followers will make. We, therefore, consider two

24 As an intermediate step we can write the standard formulation

$$
u_{i}^{s}\left(x_{1}, \ldots, x_{n}\right)=E-x_{i}+m X-\alpha_{i} \frac{1}{n-1} \sum_{j \neq i} \max \left\{0, u_{j}-u_{i}\right\}-\beta_{i} \frac{1}{n-1} \sum_{j \neq i} \max \left\{0, u_{i}-u_{j}\right\}
$$

and then substitute in contributions for monetary payoff. 
possibilities. The first possibility is that she focuses solely on the leader contribution, about which she is perfectly informed. Inequality relative to the leader's payoff is still given weight $n-1$ to reflect the $n$ members of the group (as in Equation (7)). Essentially, therefore, in focusing on inequality with the leader she assumes there will be no inequality with the other two followers. ${ }^{25}$ Given this assumption

$$
\frac{d u_{i}^{s}}{d x_{i}}=-1+m+\beta_{i} \frac{1}{n-1}
$$

for all $x_{i}<L$. She, therefore, maximizes her payoff by contributing $L$ if $\beta_{i}>(1-m)(n-1)$, and by contributing zero otherwise. The important thing to note here is that the critical level of the MPCR above which a follower will match the leader contribution is higher in a leader-follower game than follower-average game. This is because the follower is comparing her payoff to only one player compared to $n-1$ players in the follower-average game. The incentive to reduce inequality is thus diminished.

The second possibility we shall consider is that a follower tries to predict what other followers will do. Specifically, suppose she believes that each follower will contribute $L \geq F>0$ with probability $p$ and will contribute zero with probability $1-p$. Given this assumption

$$
\frac{d u_{i}^{s}}{d x_{i}}=-1+m+\beta_{i} \frac{1}{n-1}+\left(p \beta_{i}-\alpha_{i}(1-p)\right) \frac{n-2}{n-1}
$$

for all $x_{i}<F$. Again, we see that unless $p=1$ the critical level of the MPCR above which a follower will make a positive contribution is higher than in the follower-average game. We get, however, the further possibility that the follower will contribute $F<L$ and, therefore, not fully match the leader contribution. While we omit the details it is clear that the amount followers will contribute is increasing in the value of the MPCR. This difference can be crucial given that a failure to match the leader contribution has been linked to declining contributions in public good games [6].

We turn next to the contribution of a leader. We shall assume that a leader expects some amount of conditional cooperation. Specifically, she expects that if the average leader contribution is $L$ then each follower will contribute $L$ with probability $p$ and will contribute zero with probability $1-p$. For now we ignore the possibility that followers choose some fraction of $L$.

In a leader-follower game the expected payoff of a leader who chooses $L$ is

$$
u_{i}^{S}=E-L+m(L+p(n-1) L)-(1-p) \alpha_{i} L
$$

Thus, the leader maximizes her payoff by contributing the full endowment $E$ if

$$
m>\frac{1+(1-p) \alpha_{i}}{1+p(n-1)}
$$

and by contributing zero otherwise. This is a more general version of the condition given in the text. It suggests that there exists a critical value of the public good MPCR such that the leader has an incentive to contribute towards the public good. It also shows that the more inequality averse is the leader (i.e., the higher is $\alpha_{i}$ ) the higher is the MPCR below which she has an incentive to contribute zero.

25 This would be inaccurate as she can influence inequality relative to the other followers through her contribution. Nevertheless it may approximate how a follower would behave. 
Consider finally a follower-average game. In this case, we need to specify what a leader expects the other $n-2$ leaders will do. In keeping with the previous assumptions we assume she expects each other leader will contribute $E$ with probability $p$ and will contribute zero with probability $1-p$. If, therefore, she contributes $x_{i}$ the expected contribution of a conditionally cooperative follower is

$$
F=\frac{x_{i}+p E(n-2)}{n-1}
$$

This implies the following trade-off $\mathrm{f}^{26}$

$$
\frac{d u_{i}^{s}}{d x_{i}}=-1+m\left(1+\frac{p}{n-1}\right)-(1-p) \alpha_{i}+p \beta_{i} \frac{n(n-2)}{(n-1)^{2}}
$$

when $x_{i}<L$. We again obtain a critical value of the MPCR above which the leader has an incentive to contribute. However, the critical value above which a leader has an incentive to contribute is higher in a follower-average game than leader-follower game. This primarily reflects the diminished influence of the leader. In a leader-follower game the leader has the chance to influence $n-1$ followers. In a follower-average game she can only influence one follower.

\section{Author Contributions}

Both authors contributed equally in all aspects of the work on this paper.

\section{Conflicts of Interest}

The authors declare no conflict of interest.

\section{References}

1. Fischbacher, U.; Gachter, S.; Fehr, E. Are people conditionally cooperative? Evidence from a public goods experiment. Econ. Lett. 2001, 71, 397-404.

2. Kocher, M.G.; Cherry, T.; Kroll, S.; Netzer, R.J.; Sutter, M. Conditional cooperation on three continents. Econo. Lett. 2008, 101, 175-178.

3. Hermann, B.; Thoni, C. Measuring conditional cooperation: A replication study in Russia. Exp. Econ. 2009, 12, 87-92.

4. Martinsson, P.; Pham-Khanh, N.; Villegas-Palacio, C. Conditional cooperation and disclosure in developing countries. J. Econ. Psychol. 2013, 34, 148-155.

5. Keser, C.; van Winden, F. Conditional cooperation and voluntary contributions to public goods. Scand. J. Econ. 2000, 102, 23-39.

6. Fischbacher, U.; Gachter, S. Social Preferences, Beliefs, and the Dynamics of Free Riding in Public Goods Experiments. Am. Econ. Rev. 2010, 100, 541-556.

26 This comes from

$$
\begin{aligned}
u_{i}^{S}=E-x_{i}-\alpha_{i}(1-p) x_{i}-\beta_{i} p\left(\frac{n-2}{n-1}\right)\left(E-x_{i}\right)-\beta_{i} p\left(\frac{1}{n-1}\right)\left(\frac{x_{i}+p E(n-2)}{n-1}-x_{i}\right) \\
+m\left(x_{i}+p(n-2) E+p\left(\frac{x_{i}+p E(n-2)}{n-1}\right)\right) .
\end{aligned}
$$


7. Fischbacher, U.; Schudy, S.; Teyssier, S. Heterogeneous reactions to heterogeneity in returns from public goods. Soc. Choice Welf. 2012, 43, 195-217.

8. Kamei, K. From locality to continent: A comment on the generalization of an experimental study. J. Socio-Econ. 2012, 41, 207-210.

9. Isaac, R.M.; Walker, J.M. Group size effects in public goods provision: The voluntary contributions mechanism. Q. J. Econ. 1988, 103, 179-199.

10. Isaac, R.M.; Walker, J.M.; Williams, S.H. Group size and the voluntary provision of public goods: Experimental evidence utilizing large groups. J. Public Econ. 1994, 54, 1-36.

11. Goeree, J.K.; Holt, C.A.; Laury, S.K. Private costs and public benefits: Unravelling the effects of altruism and noisy behavior. J. Public Econ. 2002, 83, 255-276.

12. Falk, A.; Fischbacher, U. A theory of reciprocity. Games Econ. Behav. 2006, 54, 293-315.

13. Fehr, E.; Schmit, K.M.; A Theory of Fairness, Competition and Cooperation. Q. J. Econ. 1999, 114, $817-868$.

14. Güth, W.; Levati, M.V.; Sutter, M.; van Der Heijden, E. Leading by example with and without exclusion power in voluntary contribution experiments. J. Public Econ. 2007, 91, 1023-1042.

15. Rivas, M.F.; Sutter, M. The benefits of voluntary leadership in experimental public goods games. Econ. Lett. 2011, 112, 176-178.

16. Cartwright, E.; Gillet, J.; van Vugt, M. Leadership by example in the weak-link game. Econ. Inq. 2013, 51, 2028-2043.

17. Camerer, C. Behavioral Game Theory: Experiments in Strategic Interaction; Princeton University Press: New Jersey, NJ, USA, 2003.

18. Cartwright, E.; Patel, A. Imitation and the incentive to contribute early in a sequential public good game. J. Public Econ. Theory 2010, 12, 691-708.

19. Gächter, S.; Nosenzo, D.; Renner, E.; Sefton, M. Who Makes a Good Leader? Cooperativeness, Optimism, and Leading by Example. Econ. Inq. 2012, 50, 953-967.

20. Arbak, E.; Villeval, M.C. Voluntary leadership: Motivation and influence. Soc. Choice Welf. 2013, 40, 635-662.

21. Leipold, K.; Vetter, N.C.; Dittrich, M.; Lehmann-Waffenschmidt, M.; Kliegel, M. Individual and Developmental Differences in the Relationship between Preferences and Theory of Mind. J. Neurosci. Psychol. Econ. 2013, 6, 236-251.

22. Bolton, G.E.; Ockenfels, A. ERC: A theory of equity, reciprocity, and competition. Am. Econ. Rev. 2000, 90, 166-193.

23. Brandts, J.; Charness, G. The strategy versus the direct-response method: A first survey of experimental comparisons. Exp. Econ. 2011, 14, 375-398.

24. Fischbacher, U.; Gächter, S.; Quercia, S. The behavioral validity of the strategy method in public good experiments. J. Econ. Psychol. 2012, 33, 897-913.

25. Brandts, J.; Charness, G. Hot vs. cold: Sequential responses and preference stability in experimental games. Exp. Econ. 2000, 2, 227-238.

26. Fischbacher, U. z-Tree: Zurich toolbox for ready-made economic experiments. Exp. Econ. 2007, 10, 171-178.

27. Weber, R.A. 'Learning' with no feedback in a competitive guessing game. Games Econ. Behav. 2003, 44, 134-144. 
28. Engelmann, D.; Strobel, M. Inequality aversion, efficiency, and maximin preferences in simple distribution experiments. Am. Econ. Rev. 2004, 94, 857-869.

29. Gächter, S.; Nosenzo, D.; Renner, E.; Sefton, M. Sequential vs. simultaneous contributions to public goods: Experimental evidence. J. Public Econ. 2010, 94, 515-522.

30. Potters, J.; Sefton, M.; Vesterlund, L. Leading-by-example and signaling in voluntary contribution games: An experimental study. Econ. Theory 2007, 33, 169-182.

31. Rivas, M.F.; Sutter, M. The Dos and Don'ts of Leadership in Sequential Public Goods Experiments, No. 2008-25, Working Papers in Economics and Statistics. Available online: http://www.econstor. eu/bitstream/10419/71935/1/742551059.pdf (accessed on 7 November 2014).

32. Weber, R.; Camerer, C.; Rottenstreich, Y.; Knez, M. The illusion of leadership: Misattribution of cause in coordination games. Organ. Sci. 2001, 12, 582-598.

33. Volk, S.; Thöni, C.; Ruigrok, W. Temporal stability and psychological foundations of cooperation preferences. J. Econ. Behav. Organ. 2012, 81, 664-676.

34. Judge, T.A.; Bono, J.E. Five-factor model of personality and transformational leadership. J. Appl. Psychol. 2000, 85, 751-765.

(C) 2014 by the authors; licensee MDPI, Basel, Switzerland. This article is an open access article distributed under the terms and conditions of the Creative Commons Attribution license (http://creativecommons.org/licenses/by/4.0/). 